Sin duda, el volumen de Lema-Hincapié alcanza sus objetivos. Nos muestra un Kant distinto del de los manuales y las historias de la filosofía: un Kant que requiere matices y un seguimiento cuidadoso en cada una de las ideas, en las que confluyen el iluminismo, el criticismo, la reacción a la teología dogmática y la filosofía metafísica de su tiempo, y un enfoque moral que sin lugar a dudas tiene mucho de cristiano, aunque es difícilmente conciliable con las ortodoxias católica o protestante. Lema-Hincapié trabaja con cuidado y erudición los textos en el alemán original, revisa a los comentaristas antiguos y contemporáneos, analiza las diversas obras de Kant en paralelo, y aun del trance más difícil de su discurso - la formulación del ejercicio kantiano en terminología propia de la hermenéutica contemporánea- sale bien librado, y consigue claridad y contundencia. Si acaso pudiera formulársele algún reproche, sería el de la falta de una toma de postura personal, una evaluación propia: ¿es realmente bíblico el espíritu con el que Kant aprovecha a la Escritura? La aplicación a rajatabla del principio de pertinencia moral, ¿consigue congruencia y plenitud en la aproximación a la Biblia? ¿No es demasiado el contenido conceptual del que Kant debe prescindir como supuestamente accesorio? ¿Qué aprendizajes podría obtener la filosofía hermenéutica contemporánea —que sin duda tiene mucho de kantiana y de trascendental — de esta exégesis bíblica kantiana? Quizá dar respuesta a estas cuestiones sea demasiado pedir para un volumen de pretensiones monográficas. El texto que ofrece Andrés Lema-Hincapié merece sin duda ser considerado de referencia para aquellos interesados en el detalle del pensamiento kantiano.

VICENTE DE HARO

Facultad de Filosofía

Universidad Panamericana

vharo@up.edu.mx

Alejandro Tomasini Bassols, Filosofía y Matemáticas: ensayos en torno a Wittgenstein, Plaza y Valdés, México, 2006, 171 pp.

Considero que la filosofía de las matemáticas es de gran importancia para la filosofía en general porque aborda el estudio de una disciplina muy peculiar en cuanto que tiene múltiples usos en el mundo empírico a pesar de que, al parecer, posee principios inamovibles. Fue abordada explícitamente por Platón en la República, y tuvo un auge inusitado a fines del siglo xIx y principios del xx; época en la que se sitúan los trabajos analizados en el libro de Tomasini. El tema, sin embargo, tiene una corta historia en México y en Iberoamérica en general. De ahí la triple importancia del libro que nos ocupa: 1) se centra en algunos de los temas más relevantes y problemáticos de la filosofía de las matemáticas, motivándonos a la reflexión seria de esos temas; 2) lo hace desde el 
pensamiento de Wittgenstein, uno de los más destacados filósofos de esta área, logrando mantenerse fiel, hasta donde alcanzo a ver, al espíritu de este autor; y 3) lo hace en español, lo cual viene a traer a la mesa hispanoamericana de discusión filosófica un tema tan importante y tan descuidado. Habría que decir, además, que el autor es un gran estudioso de la obra de Russell y de Wittgenstein, y ha publicado varios libros acerca de las aportaciones de estos autores.

Tomasini inicia esta obra afirmando, con Wittgenstein, que el trabajo del filósofo de la matemática es diferente del trabajo propio de los matemáticos, ya que la filosofía, a diferencia de la matemática, no es una tarea técnica, no se trata de probar teoremas o contradicciones. Por ello, "La filosofía [...] deja todo como está. Deja también la matemática como está y ningún descubrimiento matemático puede hacerla avanzar" (Investigaciones filosóficas, § 124). Por cierto, yo mismo sostengo algo parecido en un capítulo del libro 18 Unconventional Essays on the Nature of Mathematics (Reuben Hersh, Springer, USA, 2006). Ahí defiendo que en muchas ocasiones ha habido un diálogo de sordos cuando los matemáticos y los filósofos se hacen aparentemente las mismas preguntas. En ese trabajo sostengo que cada una de esas comunidades entiende dichas preguntas de manera diferente y por ello las respuestas de unos no son significativas o satisfactorias para los otros.

A mi juicio, el libro de Tomasini es tanto una interpretación como un desarrollo del pensamiento de Wittgenstein; está compuesto de ocho capítulos que podemos agrupar en tres secciones: 1 ) dedicada a la aritmética, interpretando algunas ideas de Wittgenstein; 2) dedicada a la geometría, donde se va más allá de Wittgenstein mismo; y 3) dedicada a la concepción de Wittgenstein sobre las matemáticas en general.

\section{Sobre la aritmética}

En la sección dedicada a la aritmética (capítulos 1, 2 y 3), se presenta la concepción wittgensteniana de las matemáticas en general y, desde ahí, se propone una ontología de los números en particular. Con relación a lo primero, se afirma que los matemáticos se encargan de probar; esto es, relacionar una expresión matemática con otras expresiones matemáticas; es decir, deducen una ecuación de otras ecuaciones mediante sustituciones, ya que "no hay proposiciones matemáticas aisladas del resto" (p. 26). Por otra parte, las expresiones con las que trabajan los matemáticos no son prosa: no expresan pensamientos, "no dicen nada" (p. 27), "son un montón de cálculos", en palabras de Hintikka. En síntesis, Tomasini sostiene con Wittgenstein que los matemáticos sólo siguen reglas que ellos mismos han establecido.

Tomasini interpreta lo anterior concluyendo que "Dentro, o al interior de las matemáticas, no hay tal cosa como 'meta-matemáticas' " (p. 28), ya que ésta es prosa. A partir de eso analiza las famosas pruebas de Gödel y sostiene que, por un lado, son un montón de cálculos, y en ese sentido serían matemáticas; pero, 
por otro lado, son prosa en cuanto que afirman algo sobre la matemática. La solución de Tomasini a esa aparente dicotomía es que, aritmetizando la sintaxis, Gödel hace meta-meta-matemática. Para ello, Gödel hace primero metamatemática cuando habla desde un sistema lógico en el que pueden ser expresados enunciados acerca de la matemática. Por ejemplo, puede expresarse ahí que la aritmética es consistente o que conforma un sistema cerrado, etc. Una vez que Gödel tiene su sistema lógico, construye otro en términos aritméticos que le permite, según Tomasini, hablar sobre su sistema lógico y, por extensión, sobre la aritmética misma. Se trata de un tercer nivel; ya que si la matemática está en el primero, el sistema lógico estaría en el segundo y su traducción aritmética en el tercero.

La propuesta de Tomasini es interesante y coincido con ella en parte, aunque también encuentro problemas. En primer lugar, creo que hay que distinguir entre metamatemática y filosofía de la matemática. Esta última es, en efecto, una reflexión sobre la matemática; pero no cualquier reflexión: en palabras del propio Tomasini, se trata de "aclarar la naturaleza del número, del espacio o del infinito" (p. 12). Por otra parte, es ampliamente aceptado que la matemática y, por cierto, también la filosofía, son disciplinas recursivas. De hecho, podemos hacer matemática de la matemática, así como podemos hacer filosofía de la filosofía. Esto quiere decir que podemos hacer una reflexión filosófica sobre la filosofía misma; y, por otra parte, podemos trabajar matemáticamente los resultados matemáticos. Esto último podemos hacerlo de una forma axiomática o no.

El primer caso ocurre cuando axiomatizamos una parte de la matemática. Tal como dijo Gödel:

Las llamadas fundaciones lógicas o conjuntistas de la teoría de números, o de cualquier otra teoría matemática bien establecida, son explicativas, más bien que realmente fundacionistas, exactamente como en Física, donde la función actual de los axiomas es explicar los fenómenos descritos por los teoremas de ese sistema. ${ }^{1}$

Por otra parte, hacemos metamatemática no axiomática cuando llevamos a cabo la "transformación de una operación en elemento de un campo operacional superior: ejemplo la topología de las transformaciones topológicas". ${ }^{2}$

En esos términos, la metamatemática, axiomática o no, es matemática en cuanto que se compone de cálculos y más cálculos, según un criterio de Wittgenstein; pero también dice algo sobre la matemática misma: a saber, que los números o las operaciones se comportan entre sí de tal y cual forma. De tal manera que, por las razones anteriormente expuestas, difiero de Tomasini cuando

${ }^{1}$ I. Lakatos, Mathematics, Science and Epistemology, Cambridge University Press, Londres, 1978, p. 27.

${ }^{2}$ J. Cavailles, Méthode Axiomatique et Formalisme, Hermann Editeurs des Sciences, París, 1983, p. 173.

Diánoia, vol. LII, no. 59 (noviembre 2007). 
éste sostiene que la metamatemática no es matemática. Por supuesto, y en esto tiene razón Tomasini, la metamatemática no puede decir nada acerca de la ontología o la epistemología de dichos números u operaciones. Para esto último está la filosofía de la matemática.

Según Tomasini, Wittgenstein tiene razón en "darle la vuelta" al teorema de Gödel, en tanto que Wittgenstein no pretende hacer un trabajo matemático; es decir, técnico (p. 33). Pero Tomasini sostiene que el trabajo de Gödel no es matemático, es prosa; entonces, ¿por qué lo elude Wittgenstein? o ¿en qué sentido lo elude? podríamos preguntarnos aquí. Por otra parte, creo que tendríamos que coincidir con Tomasini cuando éste afirma que el trabajo de Gödel es un trabajo de metametamatemática; ya que si una axiomatización de la aritmética es metamatemática, el trabajo de Gödel, al hablar sobre los sistemas axiomáticos, estaría haciendo metametamatemática.

En resumen, creo que Tomasini se ve en problemas para ubicar el trabajo de Gödel desde el momento en que acepta que es metamatemático y que, por ello mismo, no es matemático. Eso le acarrea problemas para explicar qué significa que dicho trabajo sea un montón de cálculos, que sea una aritmetización y que a Wittgenstein no le interesen dichos cálculos. No obstante, si aceptamos que la metamatemática es también matemática, dichos problemas se disuelven viendo el teorema de Gödel como un trabajo matemático que habla sobre la matemática misma, por lo cual no le interesa a Wittgenstein cuando éste pretende hacer filosofía de la matemática.

En cuanto al esclarecimiento de la noción de número y su evolución en las reconocidas dos etapas de Wittgenstein, considero que Tomasini aporta algunos elementos interesantes. De entrada, Tomasini sostiene que Wittgenstein sitúa su análisis del número dentro de un estudio del lenguaje en general, defendiendo la idea de que los números no están en las cosas ni se refieren a ellas.

En la etapa del Tractatus, Wittgenstein sostiene que mientras que la totalidad de las proposiciones verdaderas es una pintura del mundo, las expresiones matemáticas no son proposiciones, ya que no dicen nada del mundo. Los números entran en las proposiciones del lenguaje ordinario sólo como cuantificadores: son el exponente de una operación, donde "una operación es aquello que hay que hacerle a una proposición para obtener otra de ella" (p. 46).

Por otra parte, en las Observaciones filosóficas, Wittgenstein afirma que "los números son retratos de extensiones de conceptos" (p. 66). Pero, como sabemos, las extensiones de los conceptos son conjuntos; y, para Wittgenstein, según Tomasini, "la idea misma de conjunto es la idea de algo conformado empíricamente" (p. 47). Pero, entonces, ¿̇cómo conciliar la idea de que las expresiones numéricas no son pinturas de algo en el mundo, con la idea de que los números son pinturas de los conjuntos empíricos asociados a los conceptos? Habría que decir que la noción de conjunto es muy problemática y Tomasini la aborda más ampliamente en la última parte. De cualquier forma, nuestro autor realiza en esta primera parte un encomiable esfuerzo en mostrar que no difieren las definiciones de número en el primero y el segundo Wittgenstein. 
Esto lo lleva a cabo mostrando que la extensión de un concepto es en realidad la manifestación de la repetición de una operación: la de aplicar el concepto a varias instancias.

De esa forma, me parece que la visión wittgensteniana de número queda esclarecida por la reflexión de Tomasini. A esto añadiría dos observaciones: 1) que el llamado segundo Wittgenstein no mantiene, a mi juicio, una división tan tajante como lo hace el primero entre las proposiciones del lenguaje ordinario y de la matemática; al parecer, en la segunda etapa ve ambas expresiones lingüísticas a partir de la idea de "reglas del juego"; y 2) en cuanto a la originalidad de Wittgenstein, hay que hacer notar que se apoya en gran medida, como él mismo lo reconoció, en la obra de Frege. Creo que fue éste quien primero vinculó el análisis de la matemática con el análisis del lenguaje en general. También fue él quien propuso que los números no estaban vinculados con las cosas empíricas, sino con cierta parte del lenguaje mediante la cual nos referimos a las cosas; es decir, estaban en otro nivel. También fue Frege quien propuso que los números formaban parte de la predicación que podemos hacer acerca de las extensiones de los conceptos mediante los que nos referimos a las cosas. Así pues, podríamos concluir que el trabajo de Wittgenstein con respecto al número se puede ver como una clarificación o perfeccionamiento, si se quiere, de la noción fregeana de número.

\section{Sobre la geometría}

En la sección dedicada a la geometría (capítulos 5 y 6), Tomasini presenta una concepción original sobre la geometría y el espacio apoyada en una revisión de autores como Kant, Poincaré, Russell, Strawson y, por supuesto, Wittgenstein. El punto de partida para Tomasini es que éstos y otros autores piensan que hay dos geometrías a las que corresponden dos nociones de espacio. La primera de ellas es axiomática y a priori, en la que el espacio y sus objetos son ideales; mientras que la otra, como rama de la física, es una ciencia empírica a posteriori que trata de los objetos preceptuales. Esta concepción se encuentra claramente expresada por autores como Poincaré y Russell.

Kant, por su parte, concibe el espacio como una condición de posibilidad de la experiencia, con lo cual, según Tomasini, Wittgenstein estaría de acuerdo; pero, de acuerdo con la perspectiva de Tomasini, Kant tiene dos fallas: 1) identificar el espacio fenomenológico y el de la física; y 2) aplicar la geometría euclidiana a ambos espacios. Como sabemos, en las teorías actuales de la física, continúa Tomasini, se aplican las geometrías no euclideanas.

En ese contexto, Wittgenstein, según Tomasini, propone que, al ser la geometría una rama de la matemática, es una sintaxis que no versa sobre nada, "sino que rige el discurso que versa sobre un sector de la realidad" (p. 117). En ese sentido, "la geometría es parte de la gramática del lenguaje, ya que fija lo que se puede decir en relación a los objetos de la percepción" (p. 103). Esta visión le parece correcta a Tomasini, aunque también sostiene que respecto de la geometría "hay diversas aseveraciones en el Tractatus de Wittgenstein que

Diánoia, vol. LII, no. 59 (noviembre 2007). 
un examen detallado muestra que no son compatibles" (p. 109). Esto se refiere a que, por un lado, se afirma que el espacio es una relación formal o necesaria entre objetos; y, por otra, que podemos tener un espacio vacío, tal y como, por cierto, lo sostenía también Newton. Pero entonces, se pregunta Tomasini: ¿cómo tener un espacio sin objetos, si el espacio es lo que justamente construyen los objetos con sus relaciones?

Desde el momento en que hay varias geometrías; es decir, la euclidiana y, al menos, las dos no euclidianas, podemos preguntarnos ¿cuál de ellas es más cercana a nuestra experiencia?, o ¿por qué algunas teorías físicas usan una versión euclidiana y otras una no euclidiana?, o, en términos de Wittgenstein, ¿por qué se usa tal o cual gramática? Tal vez, Wittgenstein diría que esta última es una pregunta sin sentido; pero pienso que algo puede decirse en relación con la geometría y creo que el mismo Witgenstein lo hizo indirectamente, como veremos más adelante.

En el libro que nos ocupa, Tomasini responde a las preguntas sobre el uso de las diversas geometrías con su propuesta de que hay tres geometrías: la pura (analítica y a priori), la perceptual (sintética a priori) y la de las teorías físicas (sintética a posteriori); a las cuales corresponden tres nociones de espacio. De esa forma, tenemos el espacio matemático completamente euclidiano; un espacio fenomenológico perceptual, al que corresponde una geometría euclidiana, aunque no totalmente; y, por último, el espacio de la física, cuya geometría es refutable.

Me parece interesante esa propuesta, pero me parece difícil sostener que haya tres y ni siquiera dos geometrías, entendiendo por éstas la pura, la empírica y la que usan las teorías físicas. A mi entender, y creo que también es la postura de Wittgenstein, la geometría es una sola y se trata de una disciplina matemática en el interior de la cual hay varias teorías axiomatizadas. Creo que la postura más defendible en este punto es la de Platón en la República, cuando afirma que las entidades matemáticas, si es que podemos hablar de tal cosa, son en todo caso entidades ideales, hipotéticas diría él; a las que pueden parecerse imperfectamente las cosas del mundo. Es así como un trío de caballos se parece al número 3, y como un cable de luz bien estirado se parece a la línea recta de Euclides. Creo que esto puede compaginarse con Wittgentein cuando éste afirma que la geometría es una disciplina incompleta: "Lo que completa a la geometría es su aplicación" (p. 104). De esta forma, tenemos, por un lado, la geometría con sus formas ideales a priori y, por otro, el parecido de estas formas ideales con las cosas del mundo empírico; es decir, la aplicación de lo ideal a lo real. Claro que Wittgenstein lo diría de otra forma; diría que las teorías físicas, por ejemplo, de la mecánica, pueden verse como mallas (redes mediante las cuales intentamos atrapar el mundo, en palabras de Popper). "A las diferentes mallas - dice Witgenstein - corresponden diversos sistemas de descripción del universo" (Tractatus, 6.341). "Pero la malla es puramente geométrica y todas sus propiedades pueden darse a priori" (6.35). De tal manera que nada dice acerca del universo que se le pueda describir auxiliado mediante cierta malla; pero "también dice algo sobre el mundo que se le pueda descri- 
bir más sencillamente por una mecánica que por otra" (Tractatus, 6.342). Lo cual lo interpreto diciendo que se han construido a priori diversas geometrías que, como gramáticas que son, rigen el discurso que versa sobre un aspecto del mundo. A ello añadiría que algunos científicos han encontrado que alguna de esas gramáticas es más apropiada que las otras para describir lo que ellos intentan describir.

\section{Sobre la matemática en general}

En la tercera sección (capítulos 4, 7 y 8), Tomasini reflexiona sobre la concepción de Wittgenstein acerca de la matemática en general. Este tema atraviesa todo el libro que nos ocupa, pero considero que Tomasini lo aborda más explícitamente en esos capítulos, si bien, como hemos visto, lo toca también para deslindar el trabajo del matemático del trabajo del filósofo de la matemática, y como marco de referencia para clasificar las pruebas de Gödel.

A juicio de Tomasini, hay filósofos realistas como Platón, Frege y Dummett, así como matemáticos realistas como Poincaré y muchos otros, que piensan que las entidades matemáticas son entidades objetivas que hay que descubrir y analizar, lo cual se lleva a cabo, según algunos realistas, mediante un tipo especial de percepción. De ahí que estos pensadores asemejen sus expresiones lingüistas a las de los físicos o biólogos cuando hablan de sus propios objetos.

La postura realista se presenta en el libro de Tomasini como un mito filosófico; pero creo que algunos pensadores realistas son rescatables. En efecto, es cierto que algunos matemáticos creen que pueden "ver" los números y otras entidades matemáticas; pero otros, aunque creen en los objetos matemáticos, los diferencian bien de los objetos de la experiencia. Frege, por ejemplo, dice que los números son objetos abstractos. Son objetivos porque no dependen de la subjetividad de quien los piensa, y son abstractos en cuanto que no están determinados espacio-temporalmente; es decir, juegan libremente en el espacio y en el tiempo, a diferencia de los objetos empíricos que están determinados espacio-temporalmente. En ese sentido, los números serían como otros objetos abstractos, tales como las notas musicales do, re, mi, etc., las cuales podemos caracterizar individualmente: la nota do, por ejemplo, y establecer relaciones bien determinadas con el resto de las notas, las cuales no están determinadas espacio-temporalmente. De manera que las expresiones musicales, así como las expresiones de la matemática, se asemejan, pero no son equivalentes, a las afirmaciones en física o en biología.

De cualquier forma, a diferencia de los realistas, Wittgenstein es para Tomasini un convencionalista o, más exactamente, un empirista convencionalista, sobre todo en su segunda etapa. Tomasini discute en esta parte con Dummett acerca de si Wittgenstein es un convencionalista, e inicia su argumentación diferenciando la visión del Tractatus de la visión del segundo Wittgenstein. En el primer caso, Tomasini sostiene que de ninguna manera podría el autor del Tractatus llamarse un convencionalista. Podemos encontrar diferencias entre la lógica y la matemática porque la primera trabaja con tautologías, mientras que

Diánoia, vol. LII, no. 59 (noviembre 2007). 
la segunda trabaja con ecuaciones; no obstante, aclara Tomasini que ambas no son pinturas de nada y exhiben, más bien, la estructura del mundo; es decir, las propiedades formales (necesarias) de las proposiciones y de los hechos que ellas representan.

Donde Wittgenstein se acerca a una posición convencionalista es en la segunda etapa, donde sostiene que el cálculo matemático es un modo de conceptualización de la realidad entre otros posibles: lo adoptamos por convención. Pero no es una convención arbitraria, ya que debe cumplir ciertos propósitos prácticos: "El cálculo es una propuesta determinada de categorización, elaborada con propósitos específicos en mente [...]. De ahí que es la praxis la que fundamenta las matemáticas" (p. 169). Es decir, tenemos la matemática que tenemos porque nos ha funcionado y, por ello, no estamos dispuestos a cambiarla. En palabras de Tomasini, "enunciamos leyes lógicas y matemáticas de manera experimental, pero una vez establecidas las volvemos inmunes a la experiencia" (p. 90). A eso, Tomasini le llama ser un empirista convencionalista. Creo que esta interpretación del convencionalismo de Wittgenstein es defendible y aporta claridad de cómo se pueden conjugar la necesidad matemática con el uso empírico de ésta.

Ahora bien, ¿dónde quedan los conjuntos en una visión como ésa? Tomasini inicia este tema destacando que la teoría de conjuntos se ha caracterizado por tener desarrollos técnicos muy importantes y cuidadosos, a la par que una confusión conceptual acerca de lo que implica la teoría misma. Esto me parece atinado ya que, al parecer, ninguna otra rama de la matemática ha suscitado tanta discusión. Frege la trató dentro de su lógica; los fundacionistas la ven como el lenguaje general de la matemática, o bien, como la parte más general y fundamental de ella. En una parte de la obra que analizamos, Tomasini le atribuye a Wittgenstein la idea de que los conjuntos están conformados empíricamente (p. 47); e, incluso que "la teoría de conjuntos no es una teoría matemática, puesto que no trabaja con números" (p. 155).

La propuesta de Tomasini con respecto a la teoría de conjuntos es que su estatus especial dentro de las matemáticas se debe a que es la gramática del lenguaje matemático, es un instrumento de otro instrumento, es la gramática del lenguaje matemático. En ese sentido, no es una teoría matemática, pero sí es una gramática, una gramática más general que la matemática misma. En ese contexto, Tomasini se pregunta “ipara qué sirve la teoría de conjuntos?” Y responde: "gracias a la teoría de conjuntos (más la lógica) se logra una efectiva aclaración conceptual de las matemáticas” (p. 154).

Esta visión es interesante y explica el papel de los conjuntos en los trabajos fundacionistas, pero creo que tiene algunos problemas. Para empezar, me parece difícil sostener hoy día que la teoría de conjuntos no sea una teoría matemática. De hecho, se compone de cálculos y más cálculos, está conectada con el resto de la matemática, sigue reglas fijadas casi convencionalmente, y creo que podemos verla como "un modo de conceptualización de la realidad entre otros posibles", usando las palabras que usa Tomasini para referirse a la matemática en general. Por otra parte, afirmar que la teoría de conjuntos no es 
una rama de la matemática porque no trabaja con números implica una concepción sobre las matemáticas que no ha sido desarrollada en este libro, y que, además, considero difícil de sostener. Si ése fuera el caso, la teoría elemental de la geometría plana, expuesta en los libros 1-4 de los Elementos de Euclides, dado que tampoco trabaja con números, tampoco sería una rama de la matemática, lo que sería difícil de sostener. Más aun, el mismo Wittgenstein afirmó en Zettel (706) que "los números no son fundamentales en matemáticas".

Para terminar, debo decir que el trabajo de Tomasini es bastante cuidadoso, pero en el capítulo dedicado a los conjuntos encontré las siguientes imprecisiones: a) en el renglón 21 de la página 142 se dice que Aleph-0 es el número de puntos de una línea, pero debería decir Aleph-1, tal y como lo expresa el mismo Tomasini unas líneas antes; b) en el renglón 4 del segundo párrafo de la página 144 se define el conjunto potencia como "el conjunto de todos los conjuntos de un conjunto dado", pero creo que sería más claro decir "el conjunto de todos los subconjuntos de un conjunto dado"; y c) en la página 146, tercer renglón del tercer párrafo, se enuncia el caso de un conjunto que supuestamente se pertenece a sí mismo: "El conjunto de todos los conjuntos de objetos que están sobre el escritorio sí es un conjunto"; pero, si lo entiendo bien, este ejemplo sólo muestra que se trata de un conjunto, no que se pertenece a sí mismo. Un ejemplo adecuado podría ser "el conjunto A de todos los conjuntos B, C, $\mathrm{D}$, E, etc., que tienen más de tres elementos". En este caso, A sí pertenece a A, y no es, por cierto, un conjunto que implique una paradoja. De cualquier forma, quiero enfatizar que, en mi opinión, estas imprecisiones no afectan la argumentación central del libro que nos ocupa, ni siquiera la correspondiente al capítulo referente a los conjuntos.*

\author{
Alfonso Ávila del Palacio \\ Instituto de Ciencias Sociales \\ Universidad Juárez del Estado de Durango \\ acavila@dgo.megared.net.mx
}

María Antonia González Valerio, El arte develado. Consideraciones estéticas sobre la hermenéutica de Gadamer, Herder, México, 2005, 170 pp.

El tema central que nos ofrece María Antonia González Valerio es el estrecho vínculo que existe entre la estética y la hermenéutica de Hans-Georg Gadamer. Mediante una comparación de la estética gadameriana con otras teorías y reflexiones estéticas que la anteceden, tales como la Crítica del juicio de Kant,

*Agradezco al Comité de Dirección de Diánoia la favorable acogida de mi reseña, así como los puntuales comentarios del árbitro anónimo que me hicieron revisar, modificar y aclarar varios puntos importantes de mi trabajo.

Diánoia, volumen LII, número 59 (noviembre 2007): pp. 232-241. 